\title{
Real-Time 3D Visualization of Temperature Distribution for Data Center Thermal Management
}

\author{
Xin Chen, Hui Zhen, Yueqi Han ${ }^{1}$, Zhaoyang Wei ${ }^{2}$, Jinong Dong ${ }^{1}$, Kaichao \\ $\mathbf{W u} \mathbf{u}^{3}$
}

\begin{abstract}
Heat management has become one of the most critical challenges to the rapid development of data centers. To address this issue, a $3 \mathrm{D}$ visualization technique is proposed in this paper to analyze and manage temperature distribution in data center rooms. At first, a room is divided into uniform grids and the temperature field is established by calculating temperature values for each cell using an inverse transfer distance weighted interpolation (ITDWI) algorithm with sensor data. Then, it visualizes these cells by voxel rendering. To improve performance, weights are pre-computed to accelerate interpolation. And two different rendering strategies are adopted to better understand temperature distribution. Our approach is deployed in an actual data center room in Chinese Academy of Sciences. By experiments, it can visualize the temperature field of the data center in real time, and the visualization results help to locate the hot spots conveniently and give a comprehensive view for thermal management.
\end{abstract}

Keywords: real-time visualization, data center management, volume rendering, spatial interpolation.

\section{Introduction}

Recent years, data centers have been greatly increased in both size and numbers. Some large data centers run up to hundreds of thousands of IT devices, consume

\footnotetext{
${ }^{1}$ X. Chen $(\bowtie)$, H. Zhen, Y. Han, J. Dong

Scientific Data Center, Computer Network Information Center, CAS, Beijing, China e-mail: chx@cnic.cn

${ }^{2}$ Z. Wei

Scientific Data Center, Computer Network Information Center, CAS

University of Chinese Academy of Sciences, Beijing, China

${ }^{3} \mathrm{~K} . \mathrm{Wu}$

Operating Support Center, Computer Network Information Center, CAS, Beijing, China
} 
magawatts of power and incur electricity bills of millions of dollars[3]. Energy consumption has become a dramatic pressure on data center management. Thus, how to reduce energy consumption and raise energy efficiency received significant attention $[4,8,10]$.

Thermal management has proved to be an effective approach to reduce energy consumption $[1,5,9,13]$. Optimized thermal management policies can decrease cooling costs and increase hardware reliability. One of the most crucial tasks of thermal management is to understand the heat airflow and discover the hot spots. Visualization of temperature distribution is an effective method to achieve this. Many attempts have been made. Most of them only visualize the temperature field in $2 \mathrm{D}$, which is insufficient to demonstrate the entire temperature space $[5,13]$. And a great number of methods adopt computational fluid dynamics (CFD), which is time-consuming and ignores the actual running state of data center. A method was proposed to visualize the temperature distribution by $3 \mathrm{D}$ particles with data extracted from sensors [7], however, the results were not easy to understand.

This paper proposed a novel method to produce a 3D visualization for thermal management. It firstly establishes the temperature field with data from sensors, and then, visualizes the temperature field. It contributes mainly on three aspects as follows.

1. Propose a practical 3D visualization frame for thermal management by modeling temperature field as a volume data set. As a result, the issue of temperature distribution visualization could be dealt with as volume rendering.

2. Propose a novel spatial interpolation method called inverse transfer distance weighted interpolation (ITDWI). It takes transfer distance into account to conform to the heat transfer rules, which makes the visualization results more reliable. Also it introduces a weight buffer to record the weights of sensors for each cell, which accelerates the interpolation significantly.

3. Design two different rendering methods for different analysis purposes. Rendering each voxel as a box using translucent color as a visual metaphor can quickly locate hot spots and benefits to understanding the overall distribution of heat flows. Correspondingly, rendering the outmost surfaces and cross sections can observe more details. The combination of these two methods is able to meet different analysis and management needs.

The remainder of the paper is organized as follows. Our proposed method is described in Section 2. Then, experimental results are discussed in Section 3, and a summary is derived in Section 4.

\section{Our Approach}

Our proposed method can be roughly divided into two steps. Firstly it establishes the temperature field with data from sensors, and then, visualizes the temperature field. At the first step, a room is divided into uniform grids, and the cells become 
the basic visual components of our method. The temperature of each cell is calculated by a proposed spatial interpolation method called ITDWI, which assumes the sensors as heat sources and limits the transfer distance of sensors. ITDWI reduces the complexity of computation and maintains the locality effectively. We also precompute the interpolation weights for each cell, which is able to further improve performance. At the second stage, two visualization methods are used simultaneously. Rendering all the cells as voxels using translucent box rendering can produce an overall view of temperature field and can also quickly locate the hot spots. While, rendering the cross sections as surfaces can analyse temperature distribution on any cross section parallel to coordinate plane. A combination of these two methods gives a comprehensive understanding of temperature distribution.

\subsection{Temperature Field Model}

To abstract the problem, we establish the model of temperature field at first. We divide the temperature field into voxels and transform our problem into a volume data issue $[2,6,11,12]$.

Data center consists of many rooms. In this paper, we use a room as a study unit. For a given room $r=(w, h, d)$, width $w$, height $h$, and depth $d$. We divide the room into a regularly spaced, 3 dimensional grids. The size of a grid cell determines the granularity of room space division, represented by $g$. The number of cells in the rooms is represented by $n, n=w^{*} h^{*} d^{*} g^{-3}$. Each cell could be considered as a voxel, and the entire room can be represented by a collection of voxels. A cell is denoted by $v_{i}, i \in\{1,2, \ldots, \mathrm{n}\}$, the room space is $V$, $V=\left\{v_{1}, v_{2}, \ldots, v_{n}\right\}$.

Temperature field describes the temperature distribution in the entire room space. It can also be denoted by the voxels $V$, and the temperature value is recorded in each cell. Furthermore, a collection of voxels with values is volume data. Therefore, temperature field is equivalent to a set of volume data, specifically the voxel set $V$. And visualizing the temperature distribution can be abstracted into a volume rendering problem, specifically to calculate and visualizing the set $V$.

To obtain actual sampling data of temperature distribution, we adopt sensors as data source. Sensors are commonly used in data center. In this paper we collect data from wireless temperature sensors. Given the number of sensors deployed in the room is $m$, and the sensor set is represented by $S$, then a sensor is denoted by $S_{i}, i \in\{1,2, \ldots, \mathrm{m}\}, S=\left\{S_{1}, S_{2}, \ldots, S_{m}\right\} . S_{i}=\left(\mathrm{x}_{i}, \mathrm{y}_{i}, \mathrm{z}_{i}, \mathrm{v}_{i}\right), \mathrm{x}_{i}, \mathrm{y}_{i}, \mathrm{z}_{i}, \mathrm{v}_{i}$ represents the location coordinate in width, height, depth, and the value of temperature respectively. 
For visualizing the volume data set $V$, the temperature value of each voxel has to be calculated at first. We achieve this by a spatial interpolation method with data from sensors $S$. The method will be introduced in the next subsection.

\subsection{Inverse Transfer Distance Weighted Interpolation}

\subsubsection{The Interpolation Method}

To get the value of each voxels is a kind of spatial interpolation problem which is very common. At first, let's have a look at our temperature field. Temperature distribution is a result of heat transfer. The voxels are both homogeneous and heterogeneous. Voxels close to each other have similar temperature value, meanwhile, voxels far away from each other are likely different. Therefore, thermal distribution is distance related. Inverse distance interpolation (IDW) is a widely used method for interpolation with a known scattered set of points and accords with the thermal distribution characteristic. The basic IDW function is showed as below.

$$
u(x)=\sum_{i=0}^{N} \frac{w_{i}(x) u_{i}}{\sum_{j=0}^{N} w_{j}(x)}, \text { where } w_{i}(x)=\frac{1}{d\left(x, x_{i}\right)^{p}}
$$

$u$ is an interpolation value at a given point $x$ based on samples $u_{i}=u\left(x_{i}\right)$, $i \in\{0,1, \ldots \mathrm{N}\}, x_{i}$ is a known point, $d$ is a given distance between $x$ and $x_{i} . N$ is the total number for known points, in our case is the number of deployed sensors. $p$ is a positive real number, called the power parameter. Greater values of $p$ assign greater influence to values closest to the interpolation point, in our experiments, $p=1$.

Nevertheless, thermal distribution in data center has some particularities of its own. Usually hundreds of servers run in a room, they are placed in large racks which become obstacles to heat transfer. Consequently, temperature distribution in data center has regional characteristic. We introduce a parameter $d t$ to denote the largest influence distance of an interpolation point (known), i.e. the transfer distanced of a sensor. The function of our inverse transfer distance weighted interpolation is as follows.

$$
t(v)=\sum_{i=0}^{N} \frac{w_{i}(v) \mathrm{t}_{i}}{\sum_{j=0}^{N} w_{j}(v)}, \text { where } w_{i}=\left\{\begin{array}{l}
\frac{1}{d\left(\mathrm{~S}_{i}, \mathrm{v}\right)^{p}}, \mathrm{~d}\left(\mathrm{~S}_{i}, \mathrm{v}\right)<=\mathrm{dt}_{i} ; \\
0, \text { otherwise }
\end{array}\right.
$$


$\mathrm{dt}_{i}$ is the transfer distance of $\mathrm{v}_{i}, t(v)$ means the temperature value of $\mathrm{v}$, $\mathrm{d}\left(\mathrm{S}_{i}, \mathrm{v}\right)$ denotes the distance between sensor $\mathrm{S}_{i}$ and voxel $\mathrm{v}$. The transfer distance of each sensor is assigned according to its location and the devices around.

In this manner, interpolation results will more close to the truth and computation complexity is also reduced.

\subsubsection{Weight Buffer}

To further improve the interpolation performance, we calculate the weights for each voxels in advance. Since the weights are only related with distance between voxel and sensors and the transfer distance of sensors, they are constant as long as the displacement of the room is not changed. We set up a buffer to record weights, the size of the weight buffer will be $n^{*} N$, which means the product of the number of voxels and the number of sensors. With the pre-computed weight buffer, weights calculation will be omitted in the real-time visualization stage, which will speed up the interpolation significantly.

Another issue should be mentioned that, the distribution of sensors usually insufficient and not optimized, therefore some voxel may be not in the transfer distance of any sensor. In this case, we calculate the most nearest $\mathrm{K}$ sensors for interpolating its value.

\subsection{Temperature Field Visualization}

Visualizing the temperature field of data center aims to help analyse and manage the heat flows. The issue concerned include whether there's a hot spot and how about the overall thermal state of the data center. To achieve this, both overall view and detail view should be produced. Therefore, we introduce two different visualizing methods for different purposes.

\subsubsection{Voxel Rendering}

We organize the temperature field as a set of volume data, therefore the temperature field can be visualized by volume rendering methods. Each voxel is assumed to be a box and will be rendered as a box. To find out the thermal distribution inside the rooms, translucent color is used to be the visual metaphor. An example of voxel rendering is showed in Fig 2.1(a).

\subsubsection{Surface Rendering}

Sometimes, thermal distribution in a cross section is needed for detail observation. To achieve this, we realize another visualization method by only rendering the 
outmost surface of the temperature field. Although it only render the outside surfaces, it's also able to render inside state just by boundary location of the temperature field. We introduce six parameters startX, startY, startZ, endX, endY, endZ to denote the boundary coordinate in 3 axes. Interactive changing the six parameters above will get different visualization result at different cross sections, which makes the understanding of thermal details easily. An example of surface rendering is showed in Fig2.1(b).

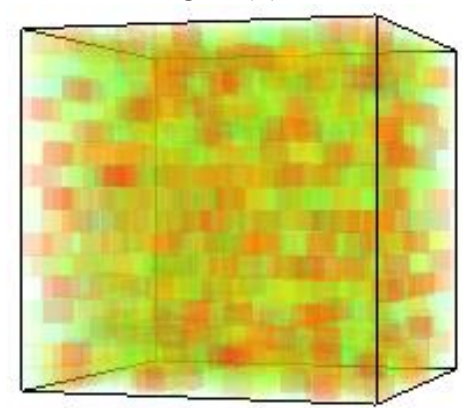

(a) voxel rendering

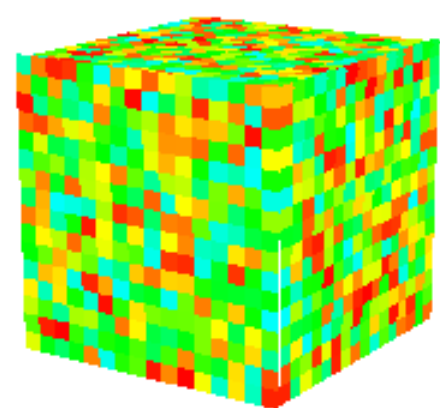

(b) surface rendering

Fig. 2.1 Two different render methods. Voxel rendering is to the left, and surface rendering is to the right. All the data of the cells are generated randomly.

\section{Results and Discuss}

The proposed method has been deployed in one room of a data center in Chinese Academy of Sciences in Beijing. Data collects from 35 sensors located in the room. The distribution and rough temperature value of these sensors are demonstrated in Fig.3.1.

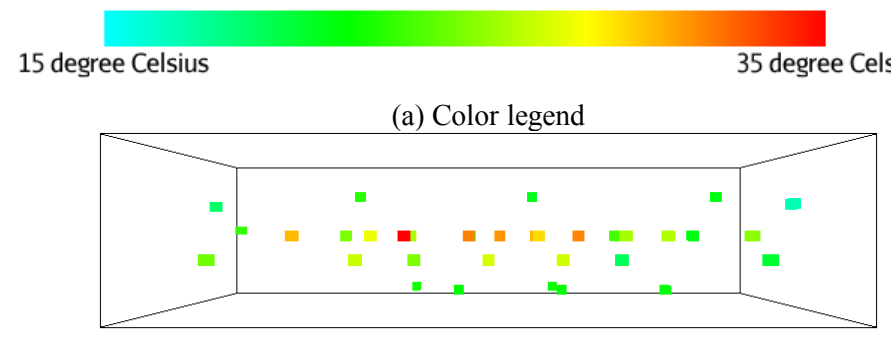

(b) Sensor distribution in our test

Fig. 3.1 There are 35 sensors deployed in the data center room. Their locations are showed in the figure. The distribution are not optimized, however, it's the real state of many data centers.

Temperature values at each voxel are computed by the ITDWI algorithm. The visualization results showed in Fig.3.2. All our experiments are implemented on a 
laptop installed with a $2.4 \mathrm{GHz}$ CPU and a integrated GPU, and the render speed can reach to more than $20 \mathrm{fps}$ when the number of voxels is about 30 thousands. It has been proved that our method is able to visualize the thermal distribution in real time.

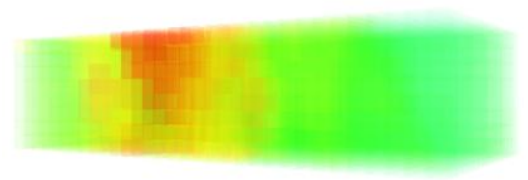

(a)

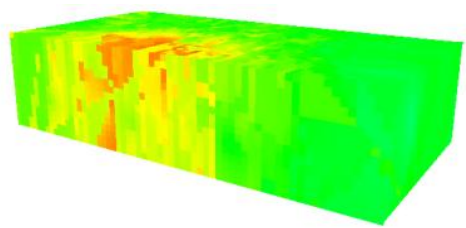

(c)

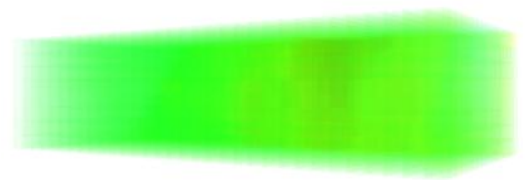

(b)

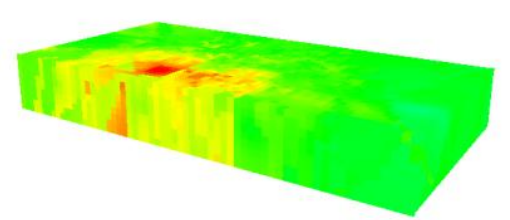

(d)

Fig. 3.2 Visualization results with data from sensors are showed above. (a) and (b) are two results of voxel rendering from different viewpoint. (c) and (d) are two surface rendering results with cross section at different heights. Figure (a) demonstrates the hot spot clearly and from figure (b) whose viewpoint is on the opposite side to that of figure (a), we can also find out the hotspot on the far end. Figure (c) and (d) give us details at different cross sections.

\section{Conclusions and Future Work}

This paper presents an effective tool for visual analytics of temperature distribution. It models the temperature field into a set of volume data. The voxel value is obtained by interpolation from sensor data using a proposed method called ITDWI. And voxel rendering and surface rendering are both adopted in our approach for visualizing, therefore, results give a more comprehensive understanding of the thermal distribution. Furthermore, an accelerating method is used for interpolation, which speeds up the calculation significantly. We deploy the proposed method in an actual running environment. Results proved that our method can real-time visualize the heat flows in $3 \mathrm{D}$ and help to analyze and manage the thermal distribution effectively.

Since both voxel calculation and voxel rendering run in parallel, the method can be easily implemented on GPU to speed up. And this method can also be used in other applications, such as humidity visualization and other environmental factors visualization indoors. Furthermore, optimized sensor distribution will give out better interpolation results. 
Acknowledgements This study is funded by Youth Foundation of Computer Network Information Center under Grant No. CNIC_QN_1304. It's also supported by Special Project of CNIC for Operations and Maintenance and Special Project of Informatization of Chinese Academy of Sciences in "the Twelfth Five-Year Plan" under Grant No. XXH12504.

\section{References}

1. Bash CE, Patel CD, Sharma RK, editors. Dynamic thermal management of air cooled data centers. Thermal and Thermomechanical Phenomena in Electronics Systems, 2006 ITHERM '06 The Tenth Intersociety Conference on; 2006 May 30 2006-June 2006.

2. Cohen I, Gordon D. VS: A surface-based system for topological analysis, quantization and visualization of voxel data. Med Image Anal. 2009 Apr;13(2):245-56.

3. Gao PX, Curtis AR, Wong B, Keshav S. It's not easy being green. ACM SIGCOMM Computer Communication Review. 2012;42(4):211-22.

4. Hamann HF, editor. A Measurement-Based Method for Improving Data Center Energy Efficiency. Sensor Networks, Ubiquitous and Trustworthy Computing, 2008 SUTC '08 IEEE International Conference on; 2008 11-13 June 2008.

5. Hao MC, Sharma RK, Keim DA, Dayal U, Patel C, Vennelakanti R. Application of Visual Analytics for Thermal State Management in Large Data Centres. Comput Graph Forum. 2010;29(6):1895-904.

6. Kruger J, Kipfer P, Kondratieva P, Westermann RD. A particle system for interactive visualization of 3D flows. Ieee T Vis Comput Gr. 2005 Nov-Dec;11(6):744-56.

7. Lange B, Rodriguez N, Puech W, Rey H, Vasques X. A 3D particle visualization system for temperature management. Proc Spie. 2011;7868.

8. Liu J, Priyantha B, Zhao F, Liang C-JM, Wang Q, James S, editors. Towards discovering data center genome using sensor nets. Proceedings of the 5th Workshop on Embedded Networked Sensors (HotEmNets); 2008.

9. Moore J, Chase J, Ranganathan P, Sharma R. Making scheduling "cool": Temperature-aware workload placement in data centers. USENIX Association Proceedings of the General Track: 2005 UNENIX Annual Technical Conference. 2005:61-74.

10. Pelley S, Meisner D, Wenisch TF, VanGilder JW, editors. Understanding and abstracting total data center power. Workshop on Energy-Efficient Design; 2009.

11. Seitz SM, Dyer CR. Photorealistic scene reconstruction by voxel coloring. Int J Comput Vision. 1999 Nov;35(2):151-73.

12. Taranukha NA, Izabekov ZA. A method for voxel visualization of 3D objects. Program Comput Soft+. 2007 Nov;33(6):336-42.

13. Xu H, Feng C, Li B, editors. Temperature aware workload management in geo-distributed datacenters. Proceedings of the ACM SIGMETRICS/international conference on Measurement and modeling of computer systems; 2013: ACM. 